\title{
Predicting the Kinetic Properties Associated with Redox Imbalance after Oxidative Crisis in G6PD-Deficient Erythrocytes: A Simulation Study
}

\author{
Hanae Shimo, ${ }^{1,2}$ Taiko Nishino, ${ }^{1,3}$ and Masaru Tomita ${ }^{1,2,3}$ \\ ${ }^{1}$ Institute for Advanced Biosciences, Keio University, 403-1, Daihoji, Tsuruoka, Yamagata 997-0017, Japan \\ ${ }^{2}$ Department of Environment and Information Studies, Keio University, Endo 5322, Fujisawa, Kanagawa 252-8520, Japan \\ ${ }^{3}$ Systems Biology Program, Graduate School of Media and Governance, Keio University, Endo 5322, Fujisawa, \\ Kanagawa 252-8520, Japan
}

Correspondence should be addressed to Masaru Tomita, mt@sfc.keio.ac.jp

Received 30 April 2011; Revised 15 June 2011; Accepted 15 June 2011

Academic Editor: Ufuk Çakatay

Copyright (๑) 2011 Hanae Shimo et al. This is an open access article distributed under the Creative Commons Attribution License, which permits unrestricted use, distribution, and reproduction in any medium, provided the original work is properly cited.

\begin{abstract}
It is well known that G6PD-deficient individuals are highly susceptible to oxidative stress. However, the differences in the degree of metabolic alterations among patients during an oxidative crisis have not been extensively studied. In this study, we applied mathematical modeling to assess the metabolic changes in erythrocytes of various G6PD-deficient patients during hydrogen peroxide$\left(\mathrm{H}_{2} \mathrm{O}_{2}-\right)$ induced perturbation and predict the kinetic properties that elicit redox imbalance after exposure to an oxidative agent. Simulation results showed a discrepancy in the ability to restore regular metabolite levels and redox homeostasis among patients. Two trends were observed in the response of redox status (GSH/GSSG) to oxidative stress, a mild decrease associated with slow recovery and a drastic decline associated with rapid recovery. The former was concluded to apply to patients with severe clinical symptoms. Low $V_{\max }$ and high $K_{\mathrm{mG} 6 \mathrm{P}}$ of G6PD were shown to be kinetic properties that enhance consequent redox imbalance.
\end{abstract}

\section{Introduction}

Glucose-6-phosphate dehydrogenase (G6PD) deficiency, an $\mathrm{X}$-chromosome linked genetic disorder, is the most prevalent mutation in humans affecting more than 400 million people worldwide [1-4]. It is characterized by the decreased activity of the G6PD enzyme, which is the central factor of the antioxidant defense system in erythrocytes (or RBCs). The enzyme is responsible for maintaining the high levels of reduced glutathione (GSH) and nicotine adenine dinucleotide phosphate (NADPH) that protect the cell from oxidative damage caused by harmful reactive oxygen species (ROS).

As RBCs are unable to generate NADPH through other pathways $[5,6]$, G6PD-deficient RBCs lack the ability to tolerate excessive amounts of oxidative stress [7-9]. The most common clinical manifestation associated with G6PD deficiency is hemolytic anemia, which is generally triggered by the intake of oxidative drugs or foods $[5,10]$. At times, the defect can result in complications such as kidney failure, severe neonatal jaundice, gallstones, and may require blood transfusion [2, 9-11].

The clinical consequences of drug-induced G6PD deficiency-related hemolysis depend on several factors including the kinetic properties of the G6PD variant. Commonly the diagnosis of patients is performed by a rapid fluorescent spot test of NADPH generation $[12,13]$ and a quantitative spectrophotometric assay of G6PD activity [10, 14]. Although these tests provide us with some perspective on the degree of severity of a patient's clinical symptoms, they are based on measurements of G6PD-specific activity when a patient is under normal conditions, and do not specifically predict the dynamic response of metabolite and enzyme activity levels in a patient's RBCs during the intake of oxidative agents. Moreover, carrying out the test during an acute hemolytic episode would give unreliable results, as the activity of the abnormal G6PD-deficient cells would be obscured by the large population of reticulocytes having relatively higher enzyme activities than the fully matured RBCs $[9,10$, 15]. Consequently, the preclinical assessment of sensitivity 
to oxidative stress in individual G6PD-deficient patients through in vivo experiments remains a difficult task [16-18]. In this study, we apply mathematical modeling to assess the changes in antioxidant enzyme activities and redox status for various individuals with G6PD deficiency during acute exposure to an oxidative agent. The main objective of this paper is to study the differences in the metabolic alterations during drug-induced hemolytic conditions in G6PDdeficient patients and predict the kinetic properties that enhance the degree of redox imbalance after an oxidative crisis.

Drug interaction in RBCs is known to produce hydrogen peroxide $\left(\mathrm{H}_{2} \mathrm{O}_{2}\right)$ directly or by way of ROS [9]. We therefore expanded an existing model of human RBC metabolism [19] to include the component reactions of ROS production and their removal processes. By inputting parameters of G6PD-deficient patients into this model, we performed simulation experiments to assess the metabolic response during exposure to oxidative $\mathrm{H}_{2} \mathrm{O}_{2}$ in patients' RBCs. We designated indicators of redox imbalance (e.g., recovery time of GSH/GSSG) from resultant simulation data to evaluate the relationship between kinetic parameters for the G6PD reaction in the patient's RBC and the patient's ability to recover from the transient oxidative perturbation. The targets of our simulation included the RBCs of 10 G6PD-deficient patients based on real data (Table 1, [21]), and virtual G6PDdeficient patients composed of randomly chosen kinetic parameters. The results of these experiments predicted the kinetic properties of G6PD that make a patient suffer major alterations in redox homeostasis after an oxidative crisis. Our simulation results provide insights for improved patientlevel treatment through the mathematical modeling and simulation of symptomatic situations, as well as a better understanding of the pathophysiology of G6PD deficiency.

\section{Materials and Methods}

The experimental workflow is presented schematically in Figure 1.

2.1. Representation of the Antioxidant System Using a Mathematical Model. A published model of human RBC metabolism [19] constructed in E-Cell System Environment Version 3 (E-Cell, $[22,23]$ ) was used as a basis for the construction of our model of the oxidative stress removal mechanism in RBCs. The model is built up of major metabolic pathways including glycolysis, pentose phosphate pathway, adenine nucleotide metabolism, glutathione metabolism, several membrane transport systems, ion leakage processes, $\mathrm{Na}^{+} / \mathrm{K}^{+}$ pump, and binding reactions of metabolites to $\mathrm{Mg}^{2+}$ and hemoglobin. The rate equations are derived from previous experimental data and are described in the Supplementary Material (see Supplementary Material available online at doi: 10.1155/2011/398945).

In the developed model (Figure 2), we incorporated the diffusion of $\mathrm{H}_{2} \mathrm{O}_{2}$ into the $\mathrm{RBC}$ and the generation of $\mathrm{O}_{2}{ }^{-}$, as well as their removal processes catalyzed by the antioxidants catalase (CAT), superoxide dismutase (SOD), and glutathione peroxidase (GSHpx). Detailed descriptions of the enzymatic equations and initial parameter values can be viewed in the Supplementary Material.

2.2. Adjustment of Kinetic Parameters to Represent Individual Patients. The reaction catalyzed by the G6PD enzyme and the reaction rate $(\nu)$ are expressed by the following equations (G6P, glucose-6-phosphate; GL6P, gluconolactone-6phosphate, 2,3BPG, 2,3-bisphosphoglycerate):

$$
\begin{aligned}
& \mathrm{G} 6 \mathrm{P}+\mathrm{NADP} \longrightarrow \mathrm{GL6P}+\mathrm{NADPH}, \\
& V_{\max } \frac{[\mathrm{NADP}][\mathrm{G} 6 \mathrm{P}]}{K_{\mathrm{mNADP}} K_{\mathrm{mG} 6 \mathrm{P}}} \\
& v=\frac{[\mathrm{NADP}]}{1+\frac{[\mathrm{G} 6 \mathrm{P}]}{K_{\mathrm{mNADP}}}}\left(1+\frac{[\mathrm{NADPH}]}{K_{\mathrm{mG} 6 \mathrm{P}}}+\frac{[\mathrm{ATP}]}{K_{\mathrm{iNADPH}}}+\frac{[2,3 \mathrm{BPG}]}{K_{\mathrm{i} 2,3 \mathrm{BPG}}},\right.
\end{aligned}
$$

where $V_{\max }$ represents the maximum initial velocity of the reaction, and $K_{m}$ and $K_{i}$ stand for the concentration of substrate that produces half-maximal velocity and inhibitor constant, respectively.

When building RBC models for simulation of individual patients, the parameters $V_{\max }, K_{\mathrm{mG} P \mathrm{P}}, K_{\mathrm{mNADP}}, K_{\mathrm{iNADPH}}$, $K_{\mathrm{iATP}}$, and $K_{\mathrm{i} 2,3 \mathrm{BPG}}$ were fit to the values specific to each patient. We first used parameter data from a previous study [21] to model 10 real G6PD-deficient patients and one healthy control. The hematological and kinetic parameters of the patients differed significantly (Table 1). Under the assumption that it would be difficult to predict the trends in the relationship between patients' kinetic parameters and their ability to restore steady state conditions after oxidative perturbation from the limited experimental data alone, we created virtual patients with these 6 parameters randomly chosen from a range of realistic values (Table 2), which ranged from the minimum to maximum values of the real patient parameter set (Table 1). Data for 500 virtual patients was obtained in each trial run.

2.3. Insertion of $\mathrm{H}_{2} \mathrm{O}_{2}$ Perturbation. To assess the dynamical response of the metabolic network of the RBCs during exposure to sudden high levels of oxidative stress, we first developed a model representing metabolic steady state and then added a perturbation of $0.1 \mathrm{mM}$ of $\mathrm{H}_{2} \mathrm{O}_{2}$ (Figure $1(\mathrm{c})$ ). The behavior over time of the ratio of reduced glutathione to oxidized glutathione (GSH/GSSG) and the ratio of reduced nicotinamide adenine dinucleotide phosphate to nicotinamide adenine dinucleotide phosphate (NADPH/NADP), as well as the enzymatic activity of G6PD, CAT, and GSHpx in each patient were investigated. We then calculated the GSH/ GSSG at steady state, the time required for GSH/GSSG to return to the normal level after the perturbation, and the amount of change in GSH/GSSG during the perturbation to evaluate the potential of oxidative stress-induced redox imbalance (Figure 1(d), [24]). Henceforth, these indicators will be referred to as "initial GSH/GSSG," "recovery time," and "amount of change," respectively. In the calculation of amount of change, we used the following equation:

Amount of change $=\frac{[\mathrm{GSH}] /[\mathrm{GSSG}]_{\mathrm{ss}}-[\mathrm{GSH}] /[\mathrm{GSSG}]_{\mathrm{min}}}{[\mathrm{GSH}] /[\mathrm{GSSG}]_{\mathrm{ss}}}$, 
TABLE 1: Hematological and kinetic parameters of G6PD-deficient patients (obtained from $[19,20]$ ).

\begin{tabular}{|c|c|c|c|c|c|c|c|c|}
\hline Patient & Reticulocytes (\%) & Half-life (days) & $V_{\max }(\mathrm{M} / \mathrm{s})$ & $K_{\mathrm{mG6P}}(\mu \mathrm{M})$ & $K_{\mathrm{mNADP}}(\mu \mathrm{M})$ & $K_{\text {iNADP }}(\mu \mathrm{M})$ & $K_{\text {iATP }}(\mu \mathrm{M})$ & $K_{\mathrm{i} 2,3 \mathrm{BPG}}(\mu \mathrm{M})$ \\
\hline Patient 1 & 40 & 2.5 & 1.1 & 152 & 3.8 & 0.62 & 180 & 520 \\
\hline Patient 2 & 2 & - & 36.7 & 140 & 115 & 33.6 & 4687 & 8515 \\
\hline Patient 3 & 2 & 22.5 & 0.8 & 7 & 4.1 & 8.9 & 952 & 1071 \\
\hline Patient 4 & 22 & - & 0.8 & 43 & 155 & 56 & 11000 & 35000 \\
\hline Patient 5 & 2 & 20.0 & 37.1 & 57 & 30.5 & 3.7 & 5016 & 5507 \\
\hline Patient 6 & 15 & 7.2 & 14.5 & 66 & 3.5 & 1.1 & 212 & 532 \\
\hline Patient 7 & 10 & - & 8.9 & 80 & 3.6 & 1 & 125 & 586 \\
\hline Patient 8 & 4 & - & 0.8 & 68 & 1.4 & 0.9 & 500 & 2000 \\
\hline Patient 9 & 30 & 5.0 & 0.6 & 40 & 4.8 & 6.9 & 314 & 3784 \\
\hline Patient 10 & 15 & - & 18.9 & 79 & 3 & 4.1 & 407 & 2200 \\
\hline Control & 1 & 27 & 64 & 67 & 3.7 & 3.1 & 749 & 2289 \\
\hline
\end{tabular}

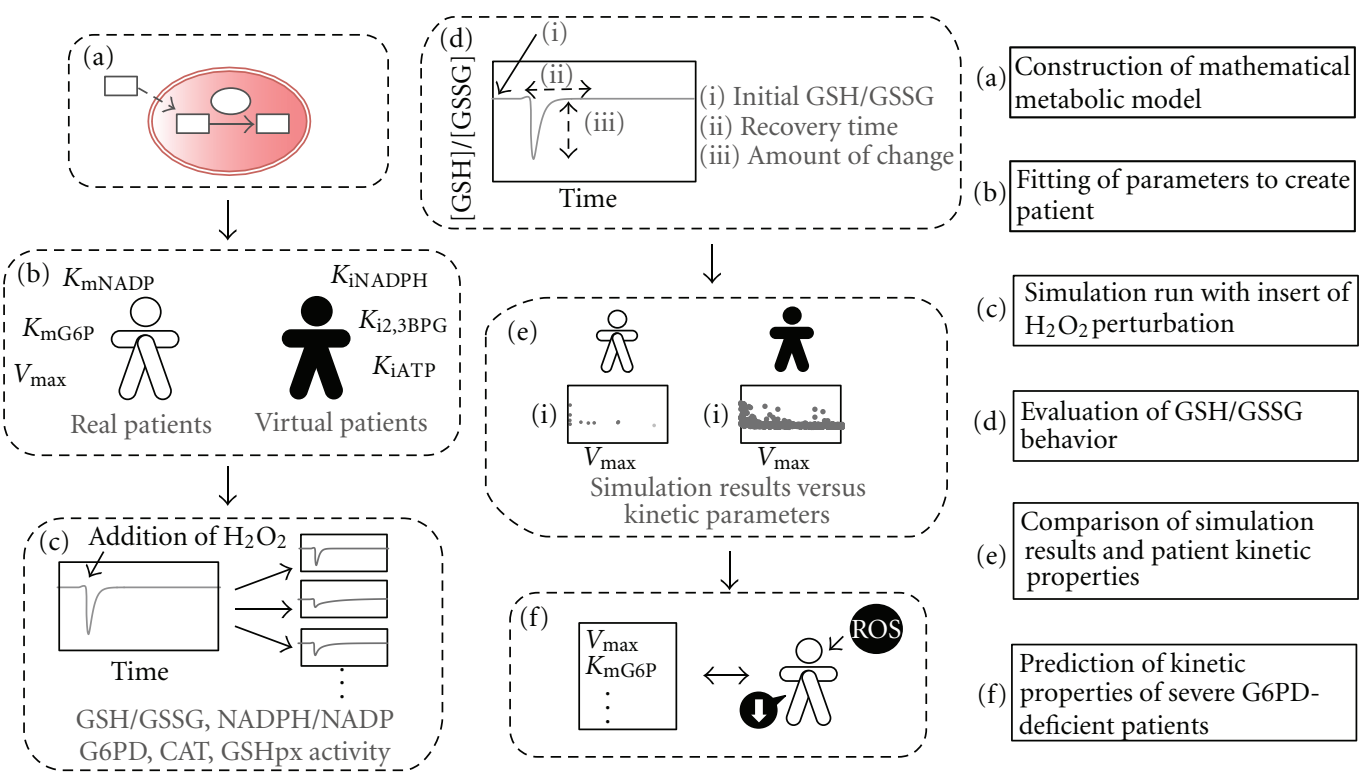

FIGURE 1: Schematic representation of the experimental workflow. (a) We first constructed a mathematical model of the human red blood cell (RBC) incorporating the antioxidant defense mechanism. (b) We then used published data from Jacobasch et al. [21] and fitted the patientspecific enzymatic kinetic parameters to build glucose-6-dehydrogenase phosphate (G6PD)-deficient patient models. Concurrently, we created virtual patient models. (c) The metabolic response of metabolite ratios and enzymatic activity to hydrogen peroxide $\left(\mathrm{H}_{2} \mathrm{O}_{2}\right)$ perturbation was simulated. (d) Recovery time and amount of change of the ratio of reduced glutathione to glutathione ratio (GSH/GSSG) were used for evaluation of patient recovering ability. (e) The relationship between kinetic parameters and the indicators of redox imbalance were examined in both real and virtual patients. (f) The kinetic properties of G6PD-deficient patients with severe symptoms were predicted from analysis results.

TABLE 2: $V_{\max }$ and kinetic constants of enzymatic reactions in virtual patients' RBCs.

\begin{tabular}{lc}
\hline Proband & Range of randomly chosen values \\
\hline$V_{\max }$ & $1-64(\mathrm{M} / \mathrm{s})$ \\
$K_{\mathrm{mG6P}}$ & $7-152(\mu \mathrm{M})$ \\
$K_{\mathrm{mNADP}}$ & $3-155(\mu \mathrm{M})$ \\
$K_{\mathrm{iNADPH}}$ & $1-56(\mu \mathrm{M})$ \\
$K_{\mathrm{IATP}}$ & $125-11000(\mu \mathrm{M})$ \\
$K_{\mathrm{i} 2,3 \mathrm{BPG}}$ & $520-35000(\mu \mathrm{M})$ \\
\hline
\end{tabular}

where $[\mathrm{GSH}] /[\mathrm{GSSG}]_{\mathrm{ss}}$ represents the initial GSH/GSSG, and $[\mathrm{GSH}] /[\mathrm{GSSG}]_{\mathrm{min}}$ represents the minimum level of
GSH/GSSG during the observation period following the perturbation.

2.4. Analysis of Simulation Data. From the simulation results, we examined the relationship of initial GSH/GSSG, recovery time and amount of change with each kinetic parameter ( $V_{\text {max }}, K_{\text {mG6P }}, K_{\text {mNADP }}, K_{\text {iNADPH }}, K_{\text {iATP }}, K_{\mathrm{i} 2,3 \mathrm{BPG}}$ ) of real G6PD patients and then performed the same analysis with the virtual patients. With these results, we plotted the values of the three indicators of redox imbalance in accordance to $V_{\max }$ and $K_{\mathrm{mG} 6 \mathrm{P}}$ in a 3 -dimensional (3D) plot to investigate the kinetic conditions that exhibit severe alterations in redox homeostasis when exposed to an oxidative agent. 


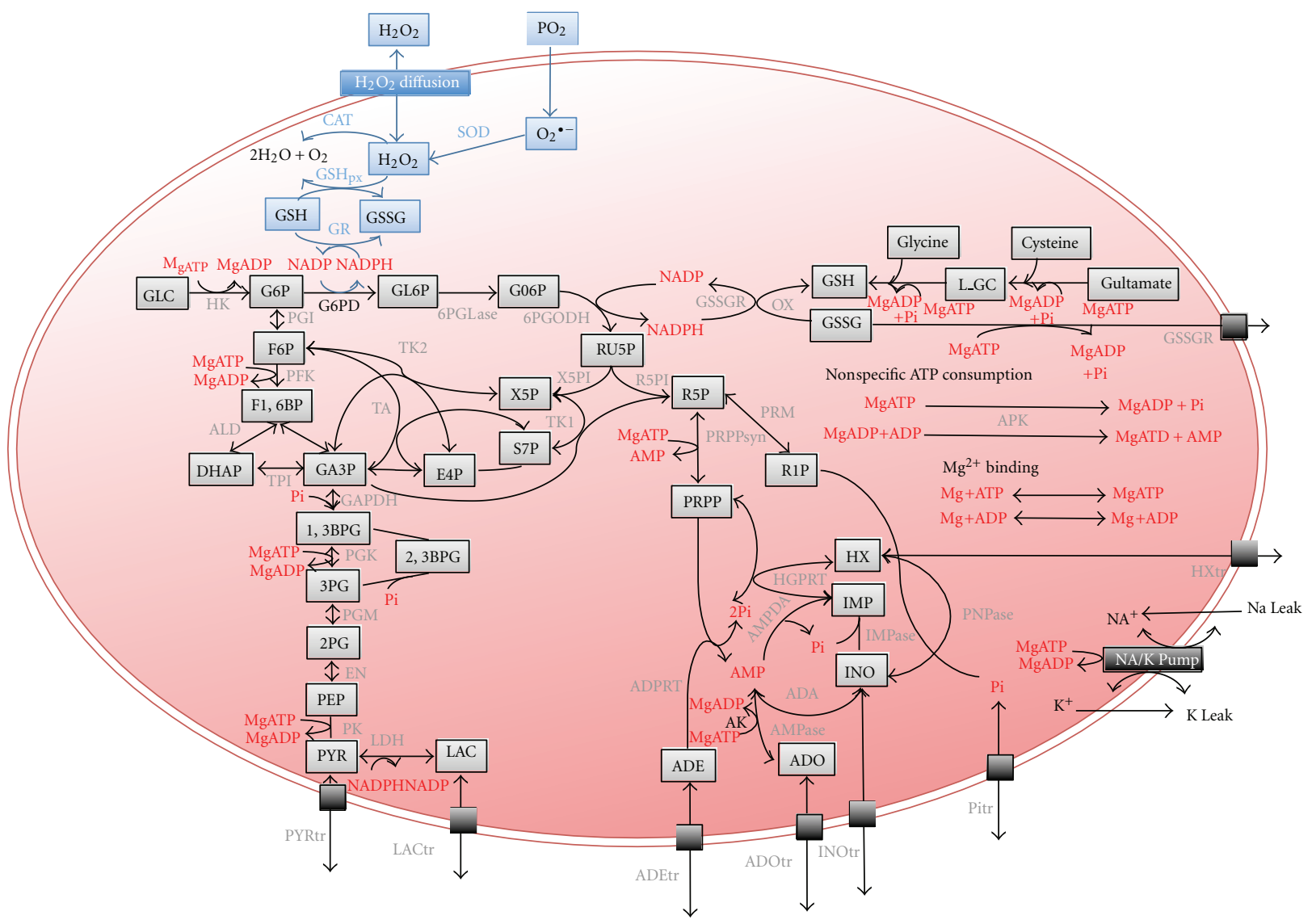

FIGURE 2: A pathway map of human red blood cell metabolism and antioxidant mechanism. The map is partially derived from Kinoshita et al. [19]. Gray boxes represent metabolites, and black boxes represent membrane transport or ion pump systems. Gray font indicates enzymes, and red font indicates the substrates or allosteric effectors of glycolytic enzymes. The blue region indicates the newly added pathway of reactive oxygen species (ROS) production and detoxification. Definitions of abbreviations for enzymes and metabolites can be found in the Supplementary Material.

\section{Results}

3.1. Metabolic Behavior of G6PD-Deficient RBCs during $\mathrm{H}_{2} \mathrm{O}_{2}$ Perturbation. The levels of GSH/GSSG and NADPH/NADP and the activity of G6PD, CAT, and GSHpx during sudden exposure to a high concentration of $\mathrm{H}_{2} \mathrm{O}_{2}$ were traced using the simulation model (Supplementary Figure 1). Substantial differences in the behavior of GSH/GSSG, NADPH/NADP, and G6PD activity in response to the input of $\mathrm{H}_{2} \mathrm{O}_{2}$ were observed among the patients. Patients 1, 4, and 8 showed the most distinctive results when compared to the control subject and other patients. The profiles of CAT and GSHpx activities were identical in all patients, but CAT activity increased to a greater degree.

A comparison of the redox status indicator GSH/GSSG in the healthy control and patient 1 is exhibited in Figure 3. The GSH/GSSG of patient 1 decreased only slightly during the oxidative perturbation; however, it took a relatively long time to return to the initial GSH/GSSG. In contrast, in the healthy control, GSH/GSSG levels dropped rapidly and returned to normal levels after a short period of time (Figure 3, Supplementary Figure 1).
3.2. Evaluation of GSH/GSSG Behavior. As mentioned previously, simulation results showed that the ability to recover from the sudden oxidative load varied among patients, in terms of recovery time and the amount of change. Therefore, in addition to initial values of GSH/GSSG, recovery time and amount of change were used to evaluate the degree of redox imbalance following oxidative stress perturbation in patients (Table 3).

The resistance of RBCs to oxidative stress did not seem to correlate with a single kinetic parameter, such as $V_{\max }$ of the G6PD reaction as can be seen in patients 3,4 , and 8 , which, despite having the same $V_{\max }$, presented distinctive recovery behavior. The healthy control had the highest initial GSH/GSSG and shortest recovery time, and patients 1, 4, and 8 who required long periods of time for recovery had the lowest initial GSH/GSSG (Table 3); patient 1 exhibited an abnormal recovery time in that the GSH/GSSG ratio did not return to the initial level during the period of observation. Overall, initial GSH/GSSG was proportional to the amount of change during the perturbation (correlation coefficient $R=0.99$ ), and recovery time was inversely proportional to amount of change ( $R=0.91$, Supplementary Figure 2$)$. 
TABLE 3: GSH/GSSG behavior of G6PD-deficient patients.

\begin{tabular}{lcccc}
\hline Proband & $V_{\max }(\mathrm{M} / \mathrm{s})$ & Initial $[\mathrm{GSH}] /[\mathrm{GSSG}]$ & Recovery time $(\mathrm{s})$ & Amount of change $($ ratio $)$ \\
\hline Patient 1 & 1.1 & 209.04 & - & 0.07 \\
Patient 2 & 36.7 & 666.76 & 279.21 & 0.19 \\
Patient 3 & 0.8 & 692.25 & 554.31 & 0.20 \\
Patient 4 & 0.8 & 388.00 & 289.74 & 0.12 \\
Patient 5 & 37.1 & 680.83 & 272.29 & 0.19 \\
Patient 6 & 14.5 & 675.73 & 286.27 & 0.19 \\
Patient 7 & 8.9 & 642.44 & 398.94 & 0.19 \\
Patient 8 & 0.8 & 515.93 & 314.17 & 0.16 \\
Patient 9 & 0.6 & 585.01 & 275.23 & 0.17 \\
Patient 10 & 18.9 & 696.04 & 235.52 & 0.20 \\
\hline Control & 64 & 700.38 & 0.20 \\
\hline
\end{tabular}

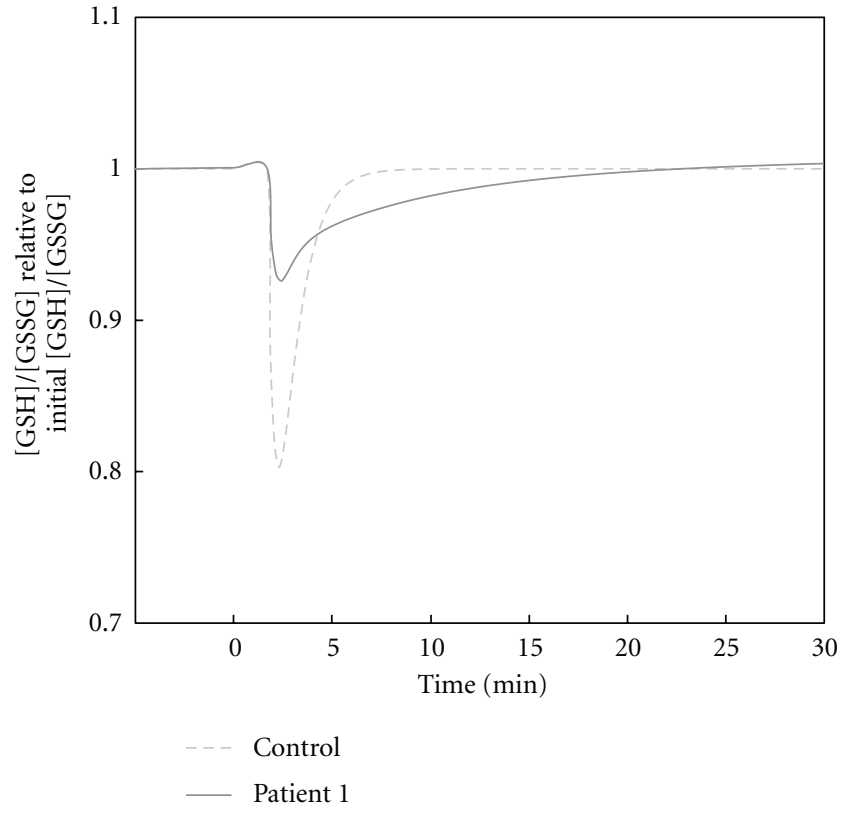

FIGURE 3: Comparison of the behavior of GSH/GSSG in the healthy control and patient 1 during $\mathrm{H}_{2} \mathrm{O}_{2}$ perturbation. $0.1 \mathrm{mM}$ of $\mathrm{H}_{2} \mathrm{O}_{2}$ was added at $0 \mathrm{~min}$, and the simulation was run for approximately $30 \mathrm{~min}$. The $y$-axis represents the ratio of [GSH]/[GSSG] relative to the initial ratio, which is set as 1.0. The figure compares the healthy subject with the patient with the longest recovery time in the set of patients (i.e., patient 1).

3.3. Effects of Kinetic Parameters on Antioxidant Capacity. Simulation results of steady state GSH/GSSG, recovery time, and amount of change were plotted against the $V_{\max }, K_{\mathrm{mG} 6 \mathrm{P}}$, $K_{\mathrm{mNADP}}, K_{\mathrm{iNADPH}}, K_{\mathrm{iATP}}$, and $K_{\mathrm{i} 2,3 \mathrm{BPG}}$ of real G6PD patients (Supplementary Figure 3); equivalent plots were constructed for virtual patients (Supplementary Figure 4). Of these 6 parameters, $V_{\max }$ and $K_{\mathrm{mG} 6 \mathrm{P}}$ showed the highest association with the indicators of redox imbalance (Supplementary Figures 3 and 4); this trend was more obvious in the data for virtual patients than real patients because of the larger number of virtual patients.

The distribution of plots for initial GSH/GSSG and amount of change were similar in both real and virtual patients (Figure 4). Most patients with high $V_{\max }$ or low $K_{\mathrm{mG6P}}$ appeared to have similarly high initial GSH/GSSG levels as well as similarly high amounts of change, whereas patients with low $V_{\max }$ and high $K_{\mathrm{mG} 6 \mathrm{P}}$, showed a broad range of values for initial GSH/GSSG and amounts of change, and a greater proportion of patients with low values for these indicators. In the virtual patients, recovery time was inversely associated with $V_{\max }$ and positively associated with $K_{\mathrm{mG} 6 \mathrm{P}}$ overall. Patients with high $V_{\max }$ or low $K_{\mathrm{mG} 6 \mathrm{P}}$ tended to have short, similar times of recovery of around $200 \mathrm{~s}$, whereas the recovery times in patients with low $V_{\max }$ or high $K_{\mathrm{mG} 6 \mathrm{P}}$ were distributed between $200 \mathrm{~s}$ to $700 \mathrm{~s}$. Several virtual patients did not conform to these trends: for example, patients with a high $V_{\max }$ but requiring a relatively long recovery time.

Simulation of virtual patient data was performed 15 times to ensure that there was no bias when determining the overall trend of the virtual patients. The degree of dispersion differed slightly between the trial runs; however, the traits given above were present in all results.

3.4. Analysis of the Relative Impact of $V_{\max }$ and $K_{\mathrm{mG} 6 \mathrm{P}}$ on the Determination of Symptom Severity. We then examined the relationship of $V_{\max }$ and $K_{\mathrm{mG} 6 \mathrm{P}}$ with initial GSH/GSSG, recovery time, and amount of change by constructing a 3-dimensional plot (Figure 5). The distribution of the graphs for initial GSH/GSSG and amount of change were very similar. When $V_{\max }$ was low $\left(V_{\max }<10\right)$, higher values of $K_{\mathrm{mG} 6 \mathrm{P}}$ were associated with lower initial GSH/GSSG, lower amount of change, and longer recovery time.

\section{Discussion}

Deficiency in G6PD activity, and hence a disturbance in redox homeostasis, can lead to severe complications during the induction of an oxidative agent if not properly diagnosed (e.g., $[25,26])$. Therefore, the preclinical assessment of the degree of metabolic dysfunction in a patient undergoing oxidative stress has been the focus of many past studies of G6PD deficiency [20, 27-29]. Although there have been several studies aiming at the mathematical representation and simulation of the metabolism in patients with G6PD deficiency [20,30-32], no studies have sought to interpret 

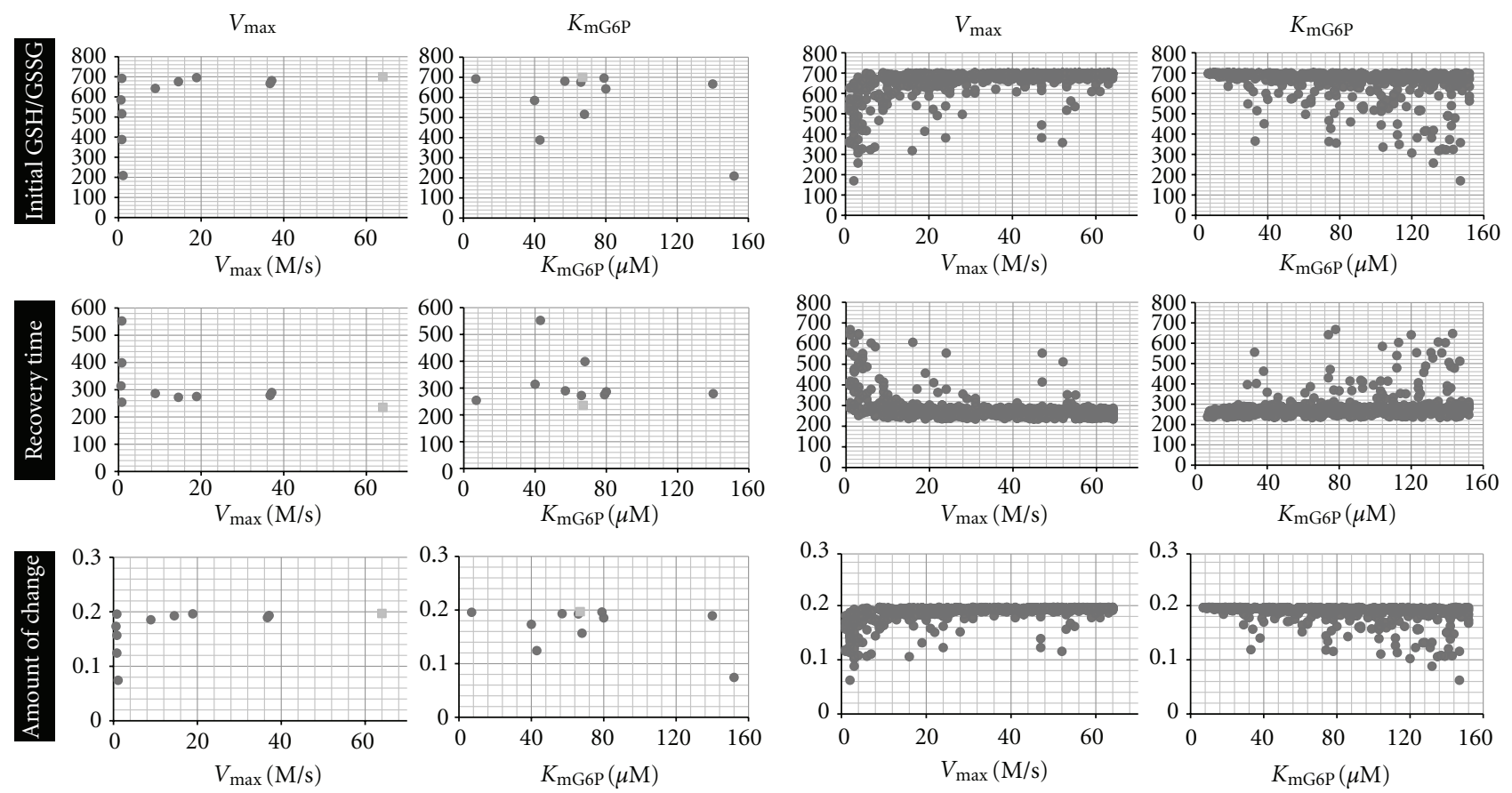

(a) Real G6PD-deficient patients
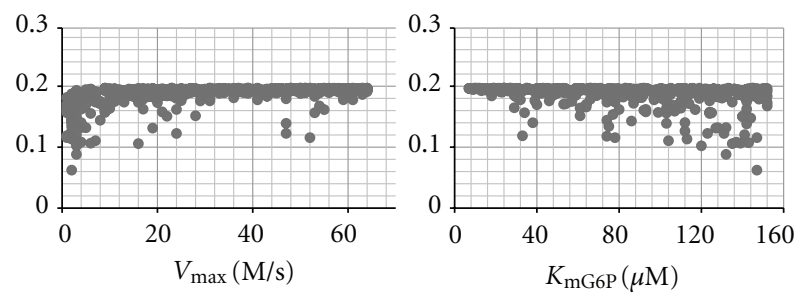

(b) Virtual patients

FIGURE 4: Comparison of G6PD kinetic parameters and steady state value, recovery time, and amount of change in GSH/GSSG in individuals. (a) The relationship between $V_{\max }$ and $K_{\mathrm{mG} 6 \mathrm{P}}$ and the evaluation criteria in real patients (Table 1). Dark gray dots represent G6PD-deficient patients, and the light gray dots represents the control. (b) The relationship between $V_{\max }$ and $K_{\mathrm{mG} 6 \mathrm{P}}$ and the evaluation criterions in virtual G6PD patients created from randomly selected parameters (Table 2). Recovery time denotes the time required for the GSH/GSSG to return to its initial value after perturbation, and amount of change is calculated by (2) in the text. Note that a few patients exhibiting abnormal recovery time ( $>30 \mathrm{~min}, 0.4 \%$ the of original number of patients) have been eliminated from the plot. The data displayed here were obtained from a single simulation run, which was representative of 15 simulation runs.

the pathways for drug-induced ROS production or determine the kinetic properties that make G6PD-deficient RBCs suffer exceptionally from critical redox imbalance after acute exposure to an oxidative agent. In this study, we examined the metabolic changes in G6PD-deficient RBCs during exposure to $\mathrm{H}_{2} \mathrm{O}_{2}$ using a model that reproduced the oxidative-stress removal mechanism and evaluated how the alterations in redox homeostasis depend on the combination of kinetic parameters for enzymatic reactions in a patient's RBCs.

The time course metabolite data showed a decrease in GSH/GSSG and NADPH/NADP, and an increase in G6PD activity during the exposure to $\mathrm{H}_{2} \mathrm{O}_{2}$ in all patients, but each to a different extent. Such behavior accurately represented the antioxidant mechanism of $\mathrm{H}_{2} \mathrm{O}_{2}$ detoxification by G6PD, resulting in depletion of GSH, rapid production of GSSG, and a subsequent decrease of NADPH, as has been shown in previous experiments [33]. The similarity in CAT and GSHpx activity among patients, independent of the degree of deficiency of the G6PD enzyme, agrees with previous observations of normal levels of antioxidant enzymes such as CAT and GSHpx in G6PD-deficient patients, independent of their hemolytic crisis history $[34,35]$. The increase in CAT and GSHpx activity in conditions where oxidative stress is sufficiently increased has also been shown in experimental data $[34,36]$. Furthermore, CAT has been recognized to have a predominant role in the removal of ROS [37], which explains the relatively high activation of CAT seen in our simulation results. Although oxidative stress-induced changes were present in the levels of all metabolites and antioxidant enzyme activity, initial levels were restored shortly after the perturbation, supporting previous observations implying that RBC metabolism is not dramatically affected by the deficiency of G6PD enzyme activity [38].

Schuster et al. [20] proposed that the severity of clinical symptoms in G6PD-deficient individuals could be assessed based on the maximal flux stimulated by an oxidative substance and upper oxidative load boundaries. However, we focused on the time-course restoration of GSH/GSSG; a redox couple that has been widely accepted as a reliable index in the estimation of cellular redox status [39]. Simulation results predicted the presence of two types of behavior in the recovery of GSH/GSSG among patients. Patients 1, 4, and 8 showed opposite traits to those of the other patients, with a change in GSH/GSSG that was relatively low in magnitude, but required long periods of time for recovery. In the control patient, GSH/GSSG dropped rapidly but recovered quickly, suggesting flexibility in the adaption to sudden change.

One might expect that oxidative stress would decrease GSH/GSSG with greater magnitude in patients with severe G6PD deficiency than those who are relatively healthy; however, in this case we found that GSH/GSSG greatlydeclined in the healthy control. The indication of sensitivity to stress 


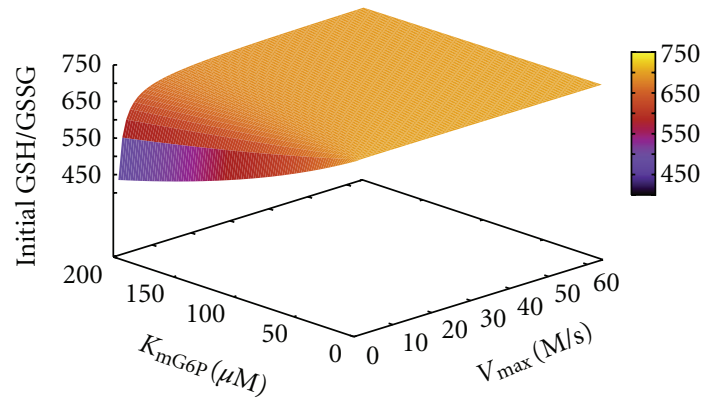

(a)

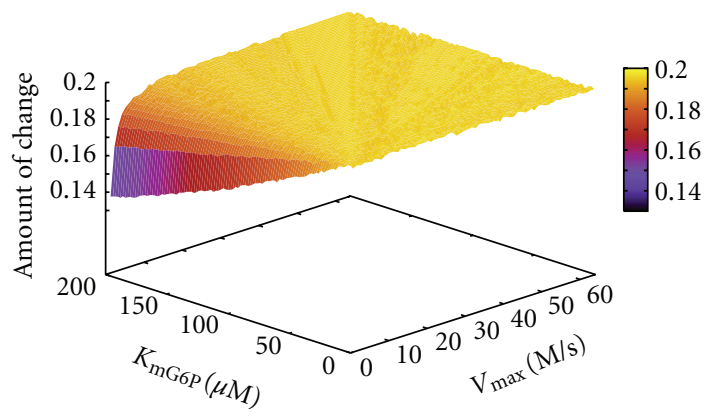

(b)

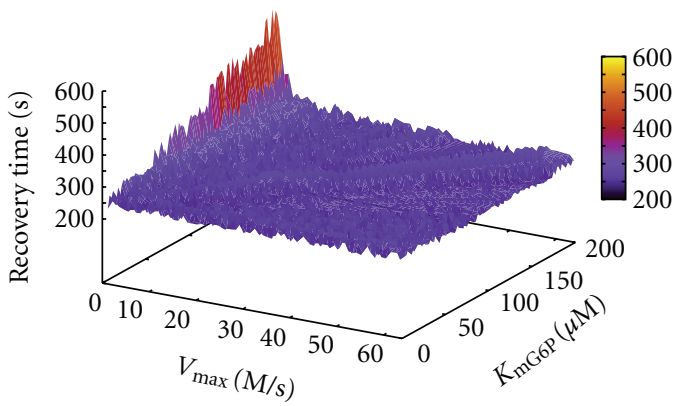

(c)

FIgURE 5: Correlation analysis of patient $V_{\max }, K_{\mathrm{mG} P}$, and GSH/GSSG behavior. The figure shows GSH/GSSG behavior under fixed values for $K_{\mathrm{mNADP}}, K_{\mathrm{iNADPH}}, K_{\mathrm{iATP}}, K_{\mathrm{i} 2,3 \mathrm{BPG}}$, and independent variables for $V_{\max }$ and $K_{\mathrm{mG} \text { ( }}$. $V_{\text {max }}$ values within the range $0-64$, and even values of $K_{\mathrm{mG} P}$ within the range 0-200, were plotted against initial GSH/GSSG (left panel), recovery time (middle panel), and amount of change (right panel).

being greater in the control patient in terms of magnitude was similar to the result of a previous report made by Arese et al. [27], who showed that oxidative divicine strongly stimulated the pentose phosphate pathway in normal RBCs, but hardly affected the metabolism in deficient cells. We speculated that the reason for the minimal amount of change of GSH/GSSG in patients 1,4 , and 8 is the patients' initial GSH/GSSG (Table 3, Figure 6). Patients 1, 4, and 8 displayed not only the longest recovery times but also the lowest initial GSH/GSSG. It is plausible that in RBCs where the initial GSH/GSSG is very low, if GSH/GSSG were to decrease greatly, the RBC could not function. We consider that the antioxidant mechanisms in the RBC may act to lessen the magnitude of GSH/GSSG reduction in such situations, and the recovery time may be lengthened as a result. Also from the accepted knowledge that cells with normal G6PD activity are highly capable of maintaining a steady GSH/GSSG [40], and redox imbalance in the RBC triggers the oxidant damage of lipids and proteins of the membrane and thus destruction of the RBC $[40,41]$, we propose that patients exhibiting a long recovery time of GSH/GSSG (i.e., severe redox imbalance) are the most severe and susceptible to hemolytic crises. Hence it can be concluded that patients 1, 4, and 8 , which exhibit long recovery time and the small initial GSH/GSSG; opposite qualities of the healthy control, are the utmost severe G6PD-deficient patients who suffer severe clinical symptoms during the intake of an oxidative substance. Although there are no clinical descriptions of patients 4 and 8 , it has been noted that patient 1 was diagnosed with severe chronic nonspherocytic hemolytic anemia [20], supporting our classification of patient 1 as a severe patient.

The expansion of simulation to include virtual patients with parameters randomly selected from a range of existing values provided a broader view of the traits in patients, simplifying the discovery of trends and enabling the prediction of susceptibility to oxidative stressin patients from their kinetic parameters (Supplementary Figure 4). The indicators of redox imbalance in patients were independent of the kinetic parameters in most graphs; however, those with $V_{\max }$ and $K_{\mathrm{mG6P}}$ as the $x$-axis, showed sign of a trait (Figure 4 ). Although patients with high $V_{\max }\left(V_{\max }>40\right)$ and those with low $K_{\mathrm{mG6P}}\left(K_{\mathrm{mG}}<\mathrm{P}<40\right)$ showed similarly high recovery abilities, as $V_{\max }$ decreased and $K_{\mathrm{mG} 6 \mathrm{P}}$ increased, the proportion of patients with low initial GSH/GSSG, long recovery time, and small amounts of change increased. These findings suggest that low $V_{\max }$ and high $K_{\mathrm{mG}}$ P enhance the likelihood of a patient having severe clinical symptoms during intake of an oxidative agent. From our observation that there were patients exhibiting short recovery time even in the low- $V_{\max }$ and high- $K_{\mathrm{mG}} \mathrm{P}$ groups, we can assume that the severity of symptoms cannot be determined by one single kinetic parameter. Also during simulation, a few virtual patients (not shown in Figure 4) exhibited abnormally long recovery times that were longer than the test period, and so had a similar recovery profile to that of patient 1 . Because we have linked a mild decrease of GSH/GSSG and long recovery time with severe disorder in redox homeostasis in patients following a hemolytic crisis, we assume that these virtual patients correspond to the most severe patients. 


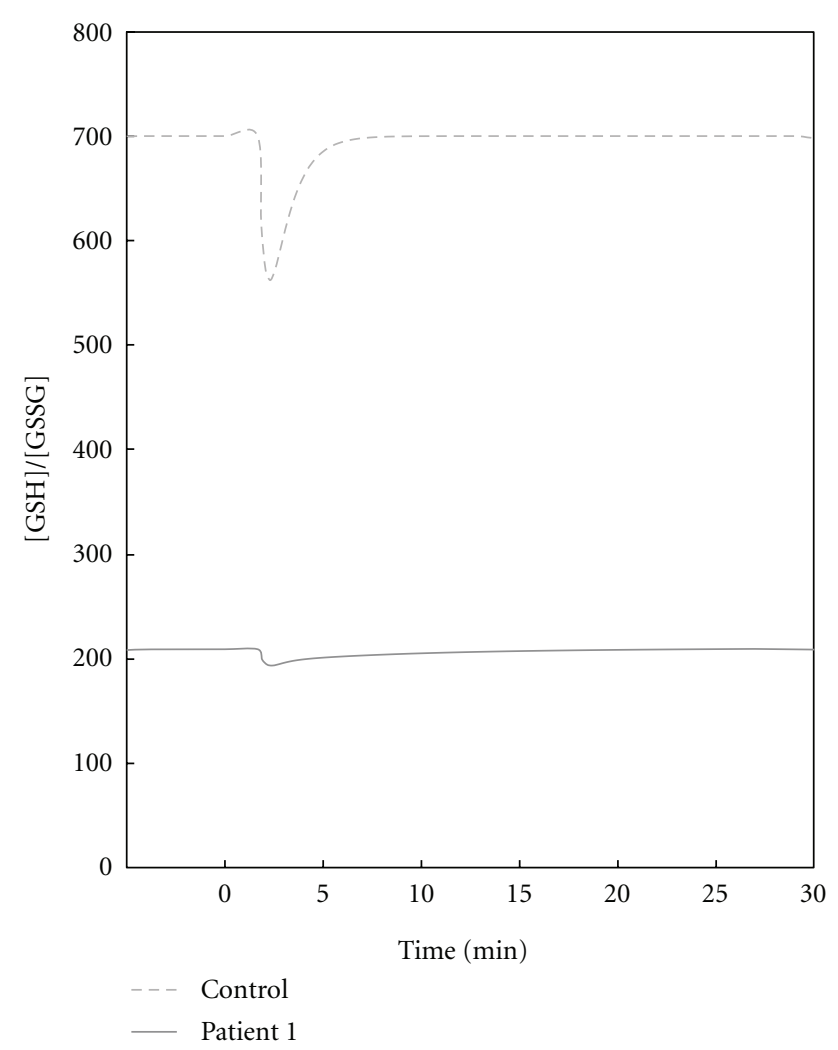

Figure 6: Comparison of time course GSH/GSSG behavior in healthy subject (above) and patient 1 (below). GSH/GSSG is given as the concentration of GSH relative to GSSG at steady state.

The results of the kinetic parameter analysis of virtual patients using a 3D image plot (Figure 5) led us to predict that in the subset of patients with low $V_{\max }$, increases in $K_{\mathrm{mG6P}}$ will be associated with increases in the severity of the patients' symptoms. These results agree with past proposals of low $V_{\max }$ and high $K_{\mathrm{mG}}$ P association with clinical severity of G6PD patients $[16,42,43]$. In fact, many patients with such properties have been classified into the most severe class of G6PD deficiency (i.e., Class I [5]) during diagnosis [16]. However, in this study, there were numerous virtual patients with low $V_{\max }$ that showed high capabilities of detoxifying oxidative stress. This contradicts the principal classification of G6PD-deficient patients [5], which mainly groups patients according to the $V_{\max }$ of the G6PD enzyme. The method of randomly choosing parameters, used here, is likely responsible for this disagreement. In this process, a combination of parameters that are not biologically feasible may have been produced, resulting in abnormal results. For example, an earlier work proposed that a low affinity to NADP may be linked to low inhibition by NADPH, ATP, and 2,3BPG [21]; however, in our simulation method, this was not taken in account.

\section{Conclusion}

In conclusion, we successfully constructed a dynamic model to represent the metabolic alterations in G6PD-deficient
RBCs during exposure to oxidative stress induced by $\mathrm{H}_{2} \mathrm{O}_{2}$. We conclude that low initial GSH/GSSG is linked with mild decrease and delay in the recovery of the steady state GSH/ GSSG after induction of an oxidative agent, and patients having this trait are susceptible to severe clinical symptoms. Furthermore, we conclude that recovering abilities do not rely on a single kinetic parameter such as $V_{\max }$. Our simulation results predicted a higher redox imbalance following oxidative damage in patients with low $V_{\max }$ and high $K_{\mathrm{mG} 6 \mathrm{P}}$. In a disorder with more than 400 biochemical variants exhibiting distinct clinical manifestations [3, 29], in which the quick screening of a large number of patients requires complicated and expensive tests that may give abnormal results $[13,44,45]$, in silico experiments such as those presented here will likely be significant for the accessible prediction of severity in pathophysiological conditions in patients without actual in vivo experimental procedures. We anticipate that our studies will also provide novel insights that will facilitate the implementation of mathematical analysis and simulation for better patient diagnosis in the future.

\section{Acknowledgments}

This work was supported by a grant from a Grant-in-Aid for Young Scientists from the Ministry of Education, Culture, Sports, Science and Technology (MEXT), CREST, and research funds from Yamagata Prefecture and Tsuruoka City to Keio University.

\section{References}

[1] E. Beutler, “G6PD deficiency," Blood, vol. 84, no. 11, pp. 36133636, 1994.

[2] J. E. Frank, "Diagnosis and management of G6PD deficiency," American Family Physician, vol. 72, no. 7, pp. 1277-1282, 2005.

[3] M. Cappellini and G. Fiorelli, "Glucose-6-phosphate dehydrogenase deficiency," The Lancet, vol. 371, no. 9606, pp. 64-74, 2008.

[4] I. Youngster, L. Arcavi, R. Schechmaster et al., "Medications and glucose-6-phosphate dehydrogenase deficiency: an evidence-based review," Drug Safety, vol. 33, no. 9, pp. 713726, 2010.

[5] E. Beutler, G. Gaetani, V. der Kaloustian, L. Luzzatto, and O. Sodeinde, "Glucose-6-phosphate dehydrogenase deficiency," Bulletin of the World Health Organization, vol. 67, no. 6, pp. 601-611, 1989.

[6] P. P. Pandolfi, F. Sonati, R. Rivi, P. Mason, F. Grosveld, and L. Luzzatto, "Targeted disruption of the housekeeping gene encoding glucose 6-phosphate dehydrogenase (G6PD): G6PD is dispensable for pentose synthesis but essential for defense against oxidative stress," EMBO Journal, vol. 14, no. 21, pp. 5209-5215, 1995.

[7] G. Jacobasch, "Biochemical and genetic basis of red cell enzyme deficiencies," Best Practice and Research, vol. 13, no. 1, pp. 1-20, 2000.

[8] G. Ronquist and E. Theodorsson, "Inherited, non-spherocytic haemolysis due to deficiency of glucose-6-phosphate dehydrogenase," Scandinavian Journal of Clinical and Laboratory Investigation, vol. 67, no. 1, pp. 105-111, 2007.

[9] E. Beutler, "Glucose-6-phosphate dehydrogenase deficiency: a historical perspective," Blood, vol. 111, no. 1, pp. 16-24, 2008. 
[10] P. J. Mason, J. M. Bautista, and F. Gilsanz, "G6PD deficiency: the genotype-phenotype association," Blood Reviews, vol. 21, no. 5, pp. 267-283, 2007.

[11] L. Luzzatto and A. Metha, "Glucose-6-phosphate dehydrogenase deficiency," in The Metabolic Basis of Inherited Disease, C. R. Scriver, A. L. Beudet, W. S. Sly, and D. Valle, Eds., pp. 33673398, McGraw-Hill, New York, NY, USA, 5th edition, 1995.

[12] E. Beutler, K. G. Blume, and J. C. Kaplan, "International Committee for Standardization in Haematology: recommended screening test for glucose-6-phosphate dehydrogenase (G-6PD) deficiency," British Journal of Haematology, vol. 43, no. 3, pp. 465-467, 1979.

[13] X. T. Gregg and J. T. Prchal, "Red cellenzymopathies," in Hematology, R. Hoffman, Ed., pp. 657-660, Churchill Livingstone, Philadelphia, Pa, USA, 4th edition, 2000.

[14] E. Beutler, "Letter: diagnosis of G.-6 P.D. deficiency," The Lancet, vol. 2, no. 7943, pp. 1032-1033, 1975.

[15] B. Ringelhahn, "A simple laboratory procedure for the recognition of A-(African type) G-6PD deficiency in acute haemolytic crisis," Clinica Chimica Acta, vol. 36, no. 1, pp. 272274, 1972.

[16] G. Jacobasch and S. M. Rapoport, "Hemolytic anemias due to erythrocyte enzyme deficiencies," Molecular Aspects of Medicine, vol. 17, no. 2, pp. 143-170, 1996.

[17] J. F. Bonilla, J. Fernando, Sanchez et al., "Glucose-6-phosphate dehydrogenase (G6PD): response of the human erythrocytes and other cells to the decrease in their activity," Colombia Médica, vol. 38, no. 1, pp. 68-75, 2007.

[18] E. Beutler, S. Duparc, O. Doumbo et al., "Glucose-6-phosphate dehydrogenase deficiency and antimalarial drug development," American Journal of Tropical Medicine and Hygiene, vol. 77, no. 4, pp. 779-789, 2007.

[19] A. Kinoshita, K. Tsukada, T. Soga et al., "Roles of hemoglobin allostery in hypoxia-induced metabolic alterations in erythrocytes: simulation and its verification by metabolome analysis," Journal of Biological Chemistry, vol. 282, no. 14, pp. 1073110741, 2007.

[20] R. Schuster, G. Jacobasch, and H. G. Holzhutter, "Mathematical modelling of metabolic pathways affected by an enzyme deficiency. Energy and redox metabolism of glucose-6-phosphate-dehydrogenase-deficient erythrocytes," European Journal of Biochemistry, vol. 182, no. 3, pp. 605-612, 1989.

[21] G. Jacobasch, D. Buckwitz, and R. Jurowski, "Heterogeneity of glucose-6-phosphate dehydrogenase enzymopathies in the GDR," Biomedica Biochimica Acta, vol. 46, no. 2-3, pp. S177S181, 1987.

[22] M. Tomita, K. Hashimoto, K. Takahashi et al., "E-Cell: software environment for whole-cell simulation," Bioinformatics, vol. 15, no. 1, pp. 72-84, 1999.

[23] A. Yachie-Kinoshita, T. Nishino, H. Shimo, M. Suematsu, and M. Tomita, "A metabolic model of human erythrocytes: practical application of the E-Cell Simulation Environment," Journal of Biomedicine and Biotechnology, vol. 2010, Article ID 642420, 14 pages, 2010.

[24] M. Asensi, J. Sastre, F. V. Pallardo et al., "Ratio of reduced to oxidized glutathione as indicator of oxidative stress status and DNA damage," Methods in Enzymology, vol. 299, pp. 267-276, 1999.

[25] M.-H. Odièvre, N. Danékova, B. Mesples et al., "Unsuspected glucose-6-phosphate dehydrogenase deficiency presenting as symptomatic methemoglobinemia with severe hemolysis after fava bean ingestion in a 6-year-old boy," International Journal of Hematology, vol. 93, no. 5, pp. 664-666, 2011.
[26] X.-J. Gu, S.-P. Chen, S.-J. Ge, L.-Q. Zheng, D.-W. Wang, and F.-X. Shen, "G6PD deficiency-induced hemolysis in a Chinese diabetic patient: a case report with clinical and molecular analysis," Acta Diabetologica. In press.

[27] P. Arese, A. Bosia, A. Naitana, S. Gaetani, M. D’Aquino, and G. F. Gaetani, "Effect of divicine and isouramil on red cell metabolism in normal and G6PD-deficient (Mediterranean variant) subjects. Possible role in the genesis of favism," Progress in Clinical and Biological Research, vol. 55, pp. 725-746, 1981.

[28] S. A. Al-Sweedan, H. Jdaitawi, W. M. Khriesat, Y. Y. Khader, and H. S. Al-Rimawi, "Predictors of severe hemolysis in patients with glucose-6-phosphate dehydrogenase deficiency following exposure to oxidant stresses," Hematology/Oncology and Stem Cell Therapy, vol. 2, no. 2, pp. 354-357, 2009.

[29] P. M. B. M. Coelho, A. Salvador, and M. A. Savageau, "Relating mutant genotype to phenotype via quantitative behavior of the NADPH redox cycle in human erythrocytes," PLoS ONE, vol. 5, no. 9, Article ID e13031, 2010.

[30] D. R. Thorburn and P. W. Kuchel, "Computer simulation of the metabolic consequences of the combined deficiency of 6-phosphogluconolactonase and glucose-6-phosphate dehydrogenase in human erythrocytes," Journal of Laboratory and Clinical Medicine, vol. 110, no. 1, pp. 70-74, 1987.

[31] T. Çakir, C. S. Tacer, and K. O. Ülgen, "Metabolic pathway analysis of enzyme-deficient human red blood cells," BioSystems, vol. 78, no. 1-3, pp. 49-67, 2004.

[32] Y. Nakayama, A. Kinoshita, and M. Tomita, "Dynamic simulation of red blood cell metabolism and its application to the analysis of a pathological condition," Theoretical Biology and Medical Modelling, vol. 2, article 18, 2005.

[33] G. F. Gaetani, C. Mareni, and E. Salvidio, "Favism: erythrocyte metabolism during haemolysis and reticulocytosis," British Journal of Haematology, vol. 43, no. 1, pp. 39-48, 1979.

[34] G. C. Gerli, L. Beretta, M. Bianchi, and A. Agostoni, "Erythrocyte superoxide dismutase, catalase and glutathione peroxidase in conditions of augmented oxidant stress," Clinical Respiratory Physiology, vol. 17, supplement, pp. 201-205, 1981.

[35] G. C. Gerli, L. Beretta, and M. Bianchi, "Erythrocyte superoxide dismutase, catalase and glutathione peroxidase in glucose6-phosphate dehydrogenase deficiency," Scandinavian Journal of Haematology, vol. 29, no. 2, pp. 135-140, 1982.

[36] S. Mueller, H. D. Riedel, and W. Stremmel, "Direct evidence for catalase as the predominant $\mathrm{H}_{2} \mathrm{O}_{2}$-removing enzyme in human erythrocytes," Blood, vol. 90, no. 12, pp. 4973-4978, 1997.

[37] G. F. Gaetani, M. Rolfo, S. Arena, R. Mangerini, G. F. Meloni, and A. M. Ferraris, "Active involvement of catalase during hemolytic crises of favism," Blood, vol. 88, no. 3, pp. 10841088, 1996.

[38] S. D. Tekir, T. Çakir, and K. O. Ulgen, "Analysis of enzymopathies in the human red blood cells by constraint-based stoichiometric modeling approaches," Computational Biology and Chemistry, vol. 30, no. 5, pp. 327-338, 2006.

[39] D. Han, N. Hanawa, B. Saberi, and N. Kaplowitz, "Mechanisms of liver injury. III. Role of glutathione redox status in liver injury," American Journal of Physiology, vol. 291, no. 1, pp. G1-G7, 2006.

[40] H.-Y. Ho, M.-L. Cheng, and D. T.-Y. Chiu, "Glucose-6-phosphate dehydrogenase-from oxidative stress to cellular functions and degenerative diseases," Redox Report, vol. 12, no. 3, pp. 109-118, 2007. 
[41] A. E. Tsantes, S. Bonovas, A. Travlou, and N. M. Sitaras, "Redox imbalance, macrocytosis, and RBC homeostasis," Antioxidants and Redox Signaling, vol. 8, no. 7-8, pp. 12051216, 2006.

[42] L. Luzzatto and U. Testa, "Human erythrocyte glucose 6-phosphate dehydrogenase: structure and function in normal and mutant subjects," Current Topics in Hematology, vol. 1, pp. 170, 1978.

[43] G. Fiorelli, F. M. di Montemuros, and M. D. Cappellini, "Chronic non-spherocytic haemolytic disorders associated with glucose-6-phosphate dehydrogenase variants," Best Practice and Research, vol. 13, no. 1, pp. 39-55, 2000.

[44] K. Iwai, H. Matsuoka, F. Kawamoto et al., "A rapid single-step screening method for glucose-6-phosphate dehydrogenase deficiency in field applications," Japanese Journal of Tropical Medicine and Hygiene, vol. 31, pp. 93-97, 2003.

[45] A. Jalloh, I. S. Tantular, S. Pusarawati et al., "Rapid epidemiologic assessment of glucose-6-phosphate dehydrogenase deficiency in malaria-endemic areas in Southeast Asia using a novel diagnostic kit," Tropical Medicine and International Health, vol. 9, no. 5, pp. 615-623, 2004. 


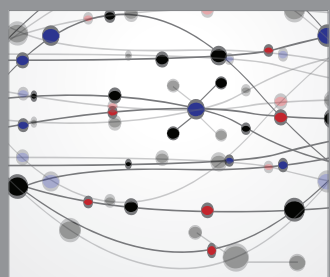

The Scientific World Journal
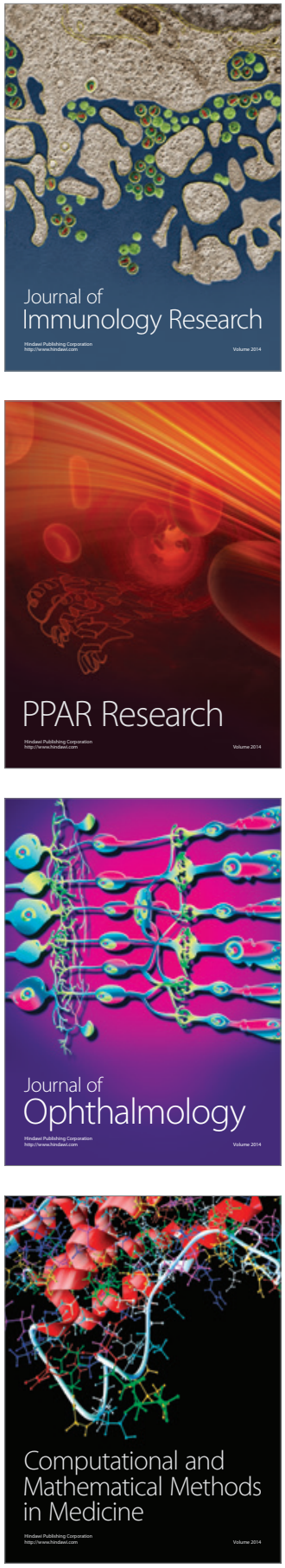

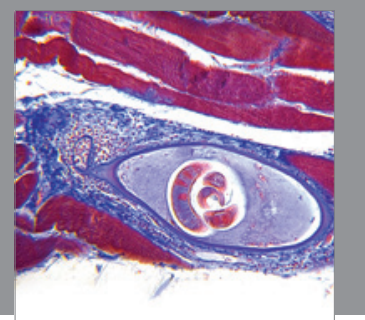

Gastroenterology

Research and Practice
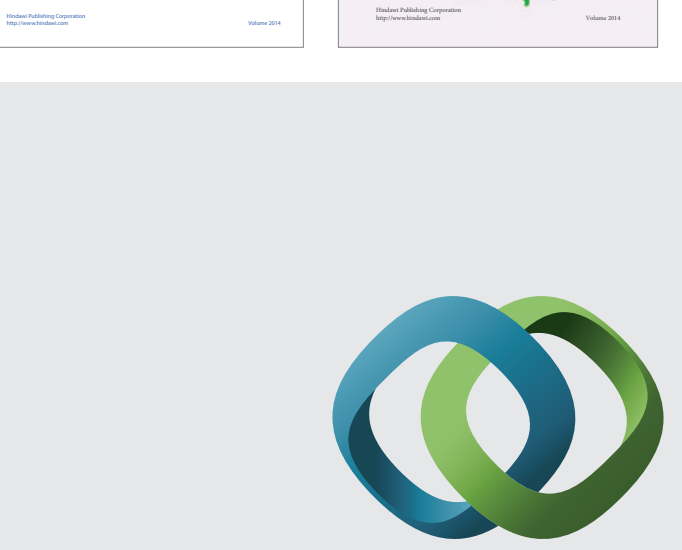

\section{Hindawi}

Submit your manuscripts at

http://www.hindawi.com
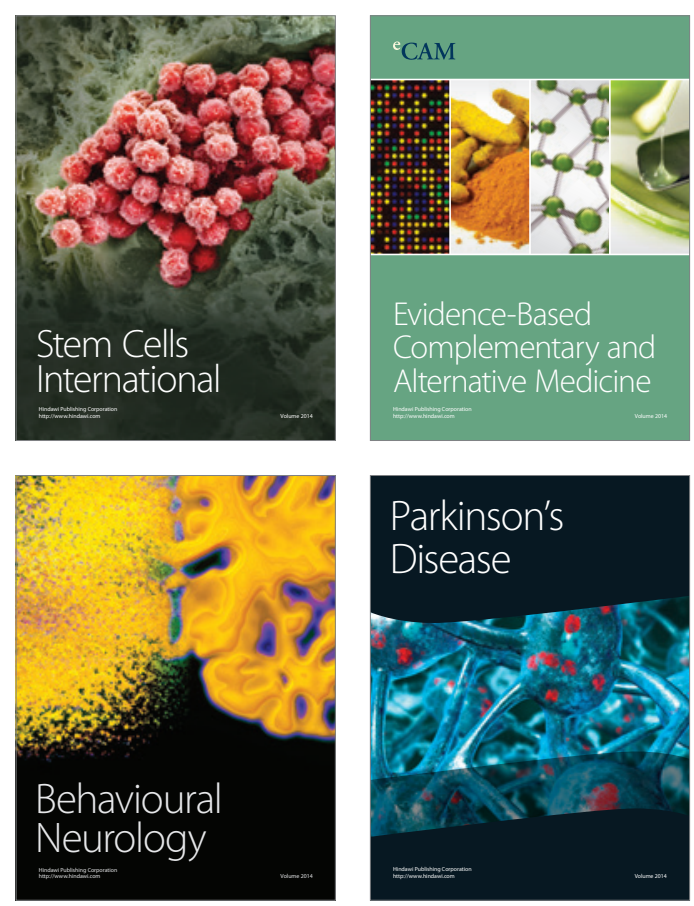

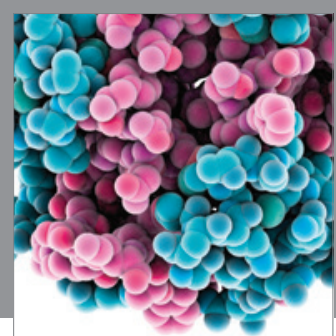

Journal of
Diabetes Research

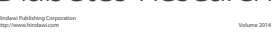

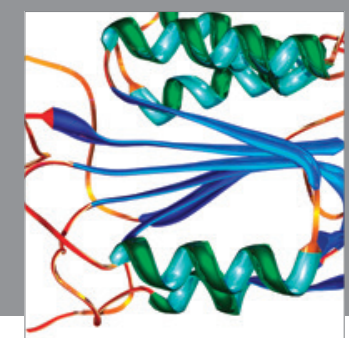

Disease Markers
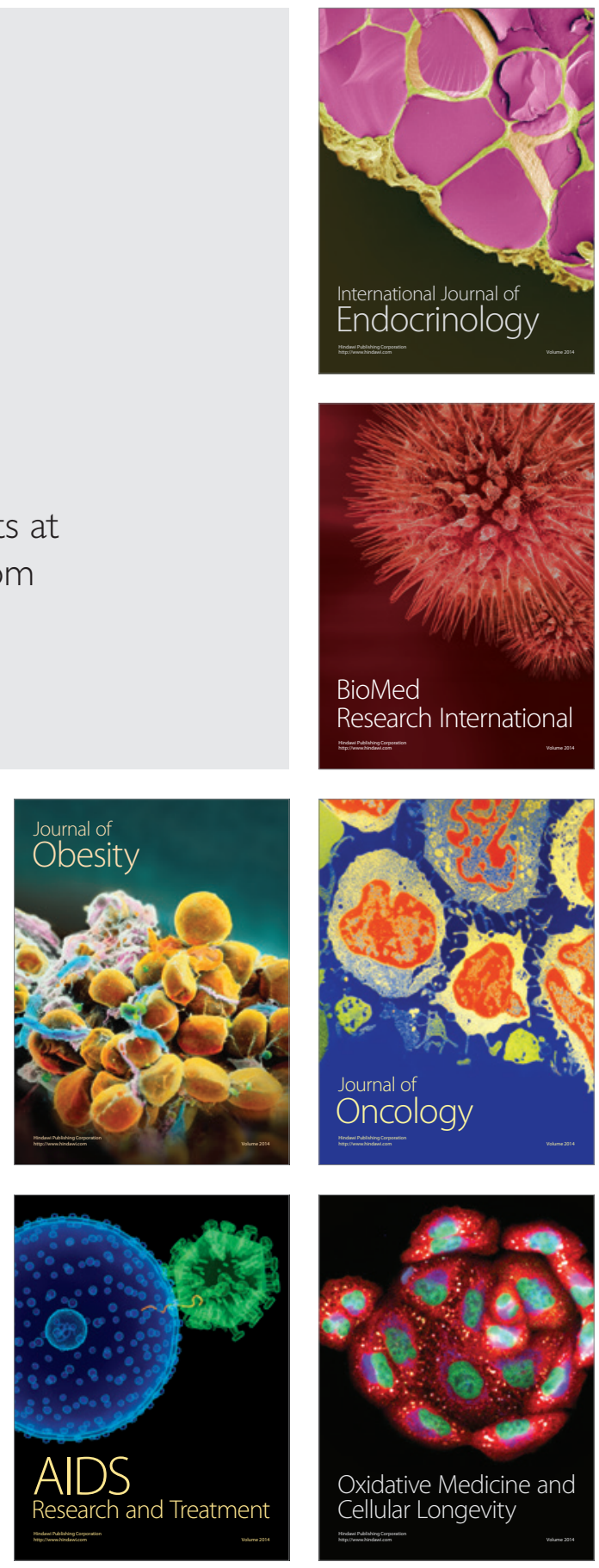